\title{
Blind Fractionally Spaced Equalization and Timing Synchronization in Wireless Fading Channels
}

\author{
Ali A. Nasir, Salman Durrani and Rodney A. Kennedy \\ School of Engineering, CECS, The Australian National University, Canberra, Australia. \\ Email: \{ali.nasir, salman.durrani, rodney.kennedy\}@anu.edu.au
}

\begin{abstract}
The development of low-complexity blind techniques for equalization and timing synchronization is of enormous importance in the design of wireless communication systems. In this paper, we propose a practical solution for blind equalization and timing recovery in fast-fading time and frequency selective wireless communication channels. We develop a general framework for Constant Modulus Algorithm (CMA) based joint Fractionally Spaced Equalization (FSE) and timing recovery. We use differential modulation to deal with any arbitrary carrier offset. We propose a data reuse strategy to achieve improved short burst wireless communication in CMA based equalization systems. Our results show that FSE outperforms $T$-Spaced Equalization (TSE) with approximately 2 times faster Mean Square Error (MSE) convergence and approximately $2 \mathrm{~dB}$ gain in Bit Error Rate (BER) performance in wireless fading channels. In addition, we demonstrate that the BER performance of the proposed FSE receiver meets the theoretical bounds with only a few dB loss in Stanford University Interim (SUI) channels, which are relevant to IEEE $802.16 .3 c$ standard for Wireless Metropolitan Area Networks.
\end{abstract}

\section{INTRODUCTION}

Blind equalization and synchronization is a very important topic for research and development in wireless communication systems [1]. Recently, there has been growing interest in blind solutions because the use of initial training sequences can reduce the date rate and may become unrealistic or impractical, especially in the context of emerging wireless ad hoc and cooperative networks which operate on opportunistic communication paradigm [2]-[6].

Blind equalization techniques can be classified into two main types. The first category is adaptive approach which is based on symbol by symbol processing and uses stochastic gradient algorithms [2]. The second category is based on batch processing and uses statistical methods, which exploit sufficient stationary statistics collected over a large block of received data [2]. A limitation of statistical methods is that they require the channel to be constant over a block of received data, which is not possible in real-world wireless fading channels. Hence stochastic gradient algorithms can be used to adapt in wireless fading channels. Constant Modulus Algorithm (CMA) is a well known stochastic gradient based algorithm because of its simplicity and ease of practical implementation [7]. CMA exploits both Fractionally Spaced Equalization (FSE) and Symbol Spaced Equalization, normally called $T$-Spaced Equalization (TSE). FSE generally performs better than TSE due to its improved time phase selectivity and global convergence under some mild conditions with zeros near the unit circle [8]. The slow convergence of CMA is well known and recently many authors have provided solutions for fast convergence in CMA [3]-[5].

So far, many researchers have proposed CMA based blind solutions for both FSE [6], [8], [9] and TSE [3]-[5]. All these papers study the mean square Error (MSE) performance of the proposed receivers under a specific channel realization and do not consider the Bit Error Rate (BER) performance in wireless fading channels. Though [10] and [11] demonstrate BER results, but the use of multiple antennas is required to achieve acceptable BER performance in wireless fading channels. Moreover, it is important to note that all the above results have been presented assuming perfect timing synchronization at the receiver, which is never possible in realistic communication systems.

In this paper, we provide a solution for constant modulus algorithm based joint FSE and timing synchronization. We employ differential modulation to deal with any arbitrary carrier offset. We propose a data reuse strategy, which exploits the adaptive nature of CMA, to achieve short block length communication by reusing the transmitted block of data to converge the estimates for equalizer weights and timing offset. We study both the MSE convergence and the BER performance and demonstrate that the proposed receiver is applicable for use in realistic time and frequency selective wireless fading channels. The major contributions of this paper, in comparison to previous research, are as follows:-

- We propose a system model for CMA based joint blind FSE and timing synchronization. The timing recovery is accomplished by using an estimator of the time derivative of the modulus of equalizer output and adjusting the timing offset in feedback mode by interpolation.

- We show that FSE outperforms TSE with 2 times faster MSE convergence and approximately $2 \mathrm{~dB}$ gain in BER performance in wireless fading channels. In addition, the proposed receiver meets the theoretical BER bounds with only a few $\mathrm{dB}$ loss under realistic SUI channels, which are relevant to IEEE 802.16.3c standard for Wireless Metropolitan Area Networks.

The rest of this paper is organized as follows. Section II describes the system model. Section III explains the proposed receiver for joint blind FSE and timing synchronization. Performance evaluation and simulation results are provided in Section IV. Finally conclusions are drawn in Section V. 


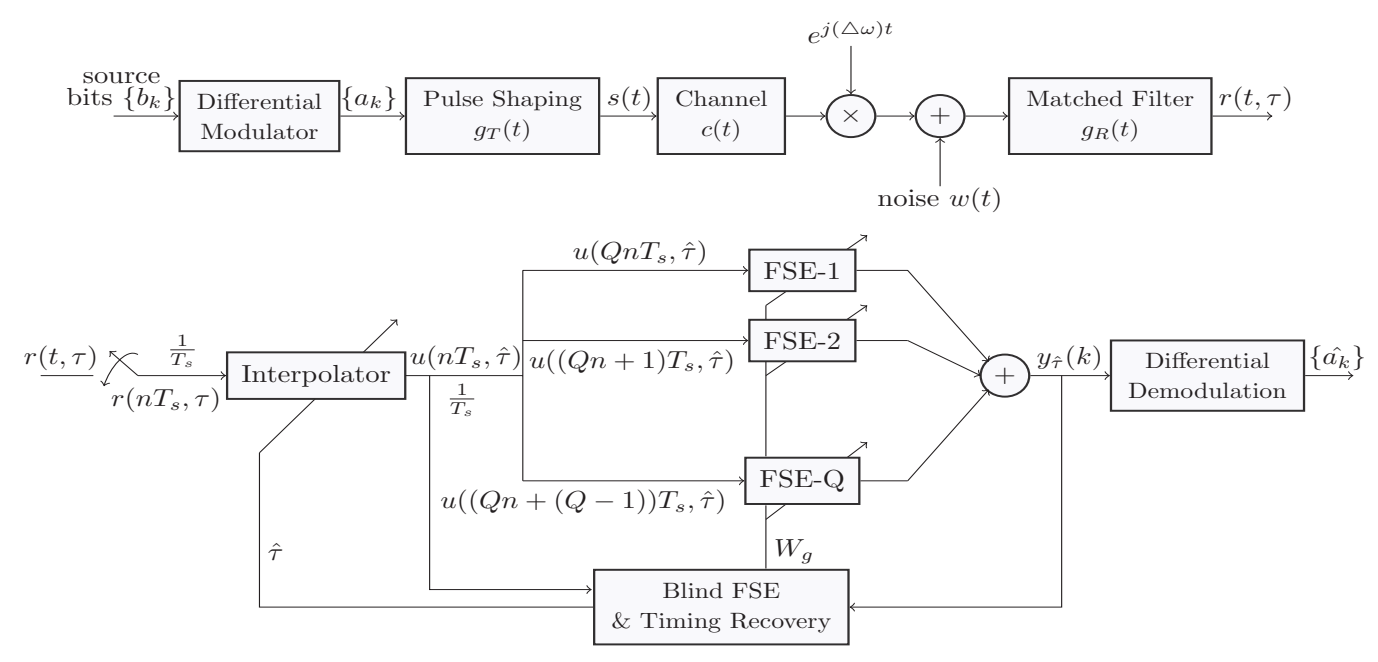

Fig. 1. Baseband Communication System for Blind Synchronization and Fractionally Spaced Equalization.

\section{SySTEM MODEL}

The baseband model of the proposed system for a single source and a single destination is shown in Fig. 1. The transmitter generates the complex valued differentially modulated sequence $a_{k}$ of data symbols, which enter the pulse shaping filter with the impulse response $g_{T}(t)$ and the resulting transmit signal $s(t)$ is given by

$$
s(t)=\sum_{k=0}^{D-1} a_{k} g_{T}(t-k T),
$$

where $1 / T$ is the symbol rate and $D$ is the number of symbols in transmission block. The signal at the output of the receive matched filter ' $g_{R}(t)$ ' is given by

$$
r(t, \tau)=\sum_{k=0}^{D-1} a_{k} h(t-\tau T-k T) e^{j \phi(t)}+v(t),
$$

where $h(t)=g_{T}(t) * c(t) * g_{R}(t)$ represents the overall baseband impulse response, $c(t)$ is the baseband multipath channel impulse response, $\phi(t)=(\Delta \omega) t$ represents the frequency offset between the transmitter and receiver oscillators, $\tau$, normalized by the symbol duration $T$, is the fractional unknown timing offset $\left(|\tau| \leq \frac{1}{2}\right)$ between the transmitter and receiver filters, $v(t)$ is the complex filtered noise $v(t)=g_{R}(t) * w(t)$ with variance $\sigma_{v}^{2}$, where $w(t)$ is the zero mean stationary, white, and complex Gaussian process and ' $*$ ' represents the convolution operator.

After pulse shaping, the signal is sampled with some timing offset since the exact sampling point corresponding with maximum Signal to Noise Ratio (SNR) is unknown for the receiver. The receive filter $r(t, \tau)$ output is oversampled by the factor $Q$ such that the oversampling period $T_{s}=T / Q$, so (2) becomes

$$
r\left(n T_{s}, \tau\right)=\sum_{k=0}^{D-1} a_{k} h\left(n T_{s}-\tau T-k T\right) e^{j \phi\left(n T_{s}\right)}+v\left(n T_{s}\right),
$$

where $n$ is the sampling index, $\phi\left(n T_{s}\right)=2 \pi(\triangle f) n T_{s}=$ $2 \pi\left(\triangle f / F_{s}\right) n, \triangle f$ is the frequency offset in $\mathrm{Hz}$ and $\triangle f / F_{s}$ is the digital frequency offset in cycles/sample [12].

\section{PROPOSED RECEIVER}

The block diagram of the receiver with joint synchronization and fractionally spaced equalization is shown in Fig. 1. The process of sampling the equalizer output at a rate $1 / T$ causes aliasing if the signal is not strictly band limited to $1 / T \mathrm{~Hz}$. Since in our case, signal is band limited to $(1+r) / T \mathrm{~Hz}$ where $r$ is the roll off factor of raised cosine pulse, a filter update at the rate $1 / T_{s}$, greater than the symbol rate, is more advantageous [13]. Thus, FSE compensates for the channel distortion in the received signal before the aliasing effects due to symbol rate sampling. While TSE exhibits local convergence problems and requires good parameter initialization for global convergence [14], FSE converges globally under some mild conditions and can be applied to the channels with zeros near the unit circle [8].

The receiver works on a symbol by symbol basis with equalizer weights and timing offset estimates being updated for each incoming symbol. The sampled output $r\left(n T_{s}, \tau\right)$ is fed to linear interpolator, which works on estimated timing offset $\hat{\tau}$ provided by the Blind FSE and Timing Recovery block. Depending on $\hat{\tau}$ and $Q$, the linear interpolation [15] between two appropriate samples is given by

$$
u\left(n T_{s}, \hat{\tau}\right)=r\left(n T_{s}, \tau\right)+\mu\left[r\left((n+1) T_{s}, \tau\right)-r\left(n T_{s}, \tau\right)\right],
$$

where $\mu=\hat{\tau} \times Q$. The sampling rate $1 / T_{s}$ is maintained after the interpolation block, i.e., interpolation is performed on every sample of the sampler output. For clarity, we reserve the index $k$ for baud-spaced i.e. $T$-spaced samples and the index $n$ for fractionally spaced samples throughout the paper.

Since FSE is fractionally spaced by the sampling factor, we use $Q$ multitap FSE branches, FSE- $(g+1)$, to mitigate ISI from multipath channel $c(t)$, where $g=0,1, \ldots, Q-1$. Let 
us denote the equalizer output $y(k T, \hat{\tau})$ as $y_{\hat{\tau}}(k)$ as shown in Fig. 1. The parameters $\hat{\tau}$ (timing offset estimate) and $W_{g}$ (the equalizer tap weight vector for $g^{\text {th }}$ FSE branch) are updated on each sample by minimizing the mean dispersion of the sampler output and using the modification in CMA cost function [5]. The modified CMA objective function can be written as

$$
J_{k}\left(\hat{\tau}, W_{g}\right)=E\left[\left(\left|y_{\hat{\tau}}(k)\right|^{2}-\gamma\right)^{2}\right],
$$

where $|\cdot|$ is the modulus operator and $E(\cdot)$ represents the expected or mean value. $\gamma$ is proportional to the kurtosis of the input sequence and is determined as [7]

$$
\gamma=\frac{E\left|a_{k}\right|^{4}}{E\left|a_{k}\right|^{2}} \text {. }
$$

Let $W_{g}(k)=\left\{w_{g, 0}, w_{g, 1}, \ldots, w_{g, N_{f}-1}\right\}^{t}$ be the adaptive tap weights of $g^{\text {th }}$ FSE at the $k^{\text {th }}$ time instant, where $N_{f}$ is the tap length of each FSE and superscript $(\cdot)^{t}$ denotes the transpose of a vector. Let the interpolated samples in the $g^{\text {th }}$ FSE delay line at the $k^{\text {th }}$ sampling time are given by

$$
X_{g}(k)=u\left((Q n+g) T_{s}, \hat{\tau}\right) \quad n=k, k-1, \ldots, k-N_{f}+1
$$

The combined output of all the FSE branches is given by

$$
y_{\hat{\tau}}(k)=\sum_{g=0}^{Q-1} X_{g}^{t}(k) W_{g}(k) .
$$

The estimated equalizer weight vectors $W_{g}(k)$ are fed to the equalizers by Blind FSE and Timing Recovery block which uses the steepest descent type method of stochastic gradient algorithm and optimizes the CMA cost function (5) to update the tap weight vectors and timing offset [2]. The steepest descent algorithm based update equations for $W_{g}(k)$ and $\hat{\tau}$ can be written as

$$
\begin{aligned}
W_{g}(k+1) & =W_{g}(k)-\mu_{w} \nabla_{w} J_{k}\left(\hat{\tau}, W_{g}\right) \\
\hat{\tau}(k+1) & =\hat{\tau}(k)-\mu_{\hat{\tau}} \nabla_{\hat{\tau}} J_{k}\left(\hat{\tau}, W_{g}\right),
\end{aligned}
$$

where $\mu_{w}$ and $\mu_{\hat{\tau}}$ are small positive step sizes which control the convergence of parameter update equations and $\nabla_{w} J_{k}\left(\hat{\tau}, W_{g}\right)$ and $\nabla_{\hat{\tau}} J_{k}\left(\hat{\tau}, W_{g}\right)$ are the gradients of the cost function (5) with respect to $g^{\text {th }}$ equalizer tap weights and timing offset respectively. Solving these gradients and then substituting in (9) and (10) results in

$$
\begin{gathered}
W_{g}(k+1)=W_{g}(k)-\mu_{w}\left(\left|y_{\hat{\tau}}(k)\right|^{2}-\gamma\right) y_{\hat{\tau}}(k) X_{g}^{H}(k), \\
\hat{\tau}(k+1)=\hat{\tau}(k)-\mu_{\hat{\tau}}\left(\left|y_{\hat{\tau}}(k)\right|^{2}-\gamma\right) \frac{\partial\left|y_{\hat{\tau}}(k)\right|^{2}}{\partial \hat{\tau}},
\end{gathered}
$$

where the superscript $(\cdot)^{H}$ represents the conjugate transpose (Hermitian) operator. The derivative of $\left|y_{\hat{\tau}}(k)\right|^{2}$ in (10) is computed as in [5].

We mitigate the effect of revolving constellation due to carrier phase offset $\phi(k)$ using differential demodulation. Finally, the differentially demodulated symbols $\hat{a_{k}}$ are decoded to produce the output information bits $\hat{b_{k}}$.

We also consider the case of $T$-symbol spaced equalization for comparison. For TSE, the set of FSE branches in Fig. 1 is replaced by a single $T$-spaced equalizer and the sampling rate after interpolation is reduced to $1 / T$, i.e., interpolation is performed on interleaved samples. As a result, the interpolator output will be written as

$$
u(k T, \hat{\tau})=r(k T, \tau)+\mu\left[r\left(k T+T_{s}, \tau\right)-r(k T, \tau)\right],
$$

where the index $k$ corresponds to baud-spaced samples. Let $W(k)=\left\{w_{0}, w_{1}, \ldots, w_{N-1}\right\}^{t}$ be the adaptive tap weights for $N$ tap single TSE and interpolated samples in the delay line of equalizer are $X(k)=\left\{u_{\hat{\tau}}(k), u_{\hat{\tau}}(k-1), \ldots, u_{\hat{\tau}}(k-N+1)\right\}^{t}$, then TSE output can be written as

$$
y_{\hat{\tau}}(k)=X^{t}(k) W(k)
$$

The equalizer taps update equation for TSE becomes

$$
W(k+1)=W(k)-\mu_{w}\left(\left|y_{\hat{\tau}}(k)\right|^{2}-\gamma\right) X^{H}(k) .
$$

\section{Simulation Results}

The simulations are carried out in MATLAB. We consider differential BPSK and QPSK modulations for system simulation. The oversampling factor $Q$ is set to 2 for implementing linear interpolation. We use root raised cosine filters truncated to $[-8 T, 8 T]$ for transmitter pulse shaping and receiver matched filtering with roll off factor $r$ set to 0.25 . The carrier frequency offset $\left(\triangle f / F_{s}\right)$ is set to a considerable value i.e. $1 / 100$ and handled by differential modulation scheme. The values of the step sizes $\mu_{\hat{\tau}}$ and $\mu_{w}$ are set to $5 \times 10^{-3}$ and $10^{-2}$ respectively.

\section{A. MSE Performance in Frequency Selective Channels}

Mean Square Error (MSE) is defined as the average instantaneous squared error between the equalizer output and the reference transmitted symbol over 80 realizations. We illustrate the MSE performance by considering one realization of multipath channel impulse response, which is also considered in [4] and [16], as

$C(z)=\left(0.4-0.6 z^{-1}+1.1 z^{-2}-0.5 z^{-3}+0.1 z^{-4}\right) e^{j \pi / 4} / 1.41$

We consider Differential QPSK (DQPSK) modulation with SNR set to $20 \mathrm{~dB}$. The timing phase offset ' $\tau$ ' is set to -0.3 . $N$ and $N_{f}$ are set to 7 i.e. the TSE ' $W$ ' and FSE-1 ' $W_{0}$ ' are selected as 7 tap filters with central tap initialized to 1 , however FSE-2 ' $W_{1}$ ' is initialized with all taps set to zero [8].

The results are shown in Fig. $2 \& 3$ for TSE and FSE respectively. Fig. 2(a) \& 3(a) shows the distorted channel output due to ISI while Fig. 2(b) \& 3(b) shows the equalized output. The circular appearance of the equalizer output is due to carrier frequency offset which is handled by differential modulation afterwards, however equalization is guaranteed by the eye opening of the equalizer output. MSE performance and estimated timing offset $\hat{\tau}$ are shown in Fig. 2(c), 2(d) and Fig. 3(c), 3(d) respectively. It can be seen that $\hat{\tau}$ converges to +0.3 approx. to mitigate the introduced negative offset. The MSE converges to an acceptable level of $-40 \mathrm{~dB}$ after just 200 symbols for FSE as compared to 450 symbols for TSE due to faster filter update rate as mentioned in Sec. III. 
(a)

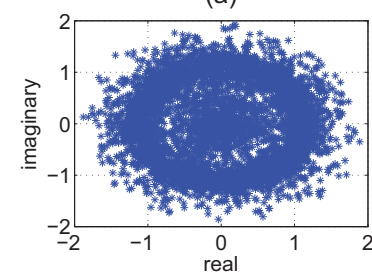

(c)
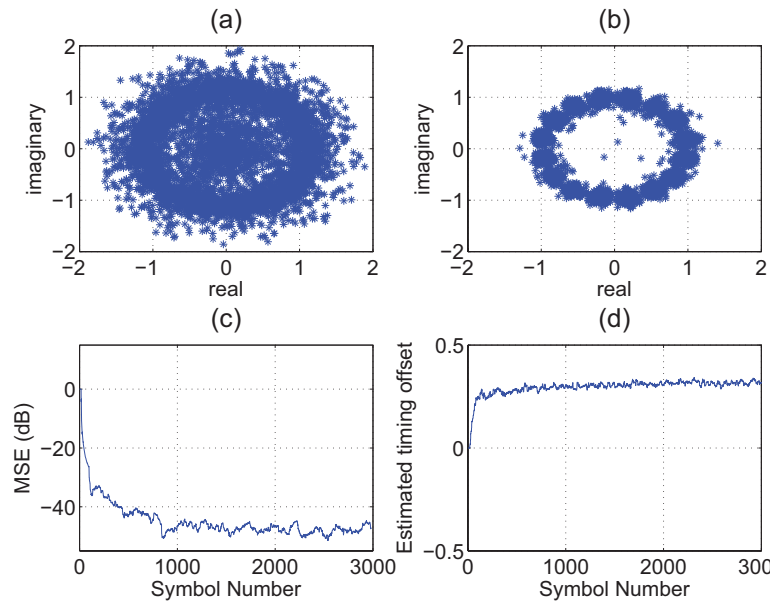

(d)

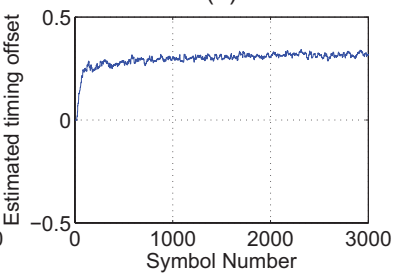

Fig. 2. Results for TSE with multipath channel, fractional timing phase offset $(\tau)=-0.3$, frequency offset $\left(\triangle f / F_{s}\right)=10^{-2}$, SNR $=20 \mathrm{~dB}$, DQPSK and equalizer length $N=7$. (a) Channel output constellation. (b) Equalized output constellation. (c) MSE between equalizer output and transmitted symbols. (d) Timing offset recovery.

\section{B. BER performance in Rayleigh and Rician fading Channels}

In this subsection, we evaluate BER performance of our system under time and frequency selective Rayleigh and SUI channels.

1) Data Reuse Strategy: In order to use the system in a wireless fading environment, we make use of data reuse strategy. It exploits the adaptive nature of CMA, where equalizer weights and timing offset estimates are updated for every incoming sample. Thus we can achieve short block length communication by reusing the transmitted block of data to converge the estimate for unknown parameters. A similar idea but in another context is presented in [11]. We achieve this by using a buffer after sampling, which stores the incoming data block in a buffer of $\beta \times D \times Q$ length by replicating the received burst $\beta-1$ times in the buffer, where $\beta$ is the data reuse factor and depends on the block length $D$ and convergence speed. Since the results for our receiver in Fig. 2 and 3 show fast convergence, we use $\beta=3$, when block length $D$ is 400 in our simulation. Demodulator ignores the first $(\beta-1) \times D$ equalized samples and demodulate on the rest of samples to decode the transmitted information.

2) Time selective Rayleigh Fading Channel: We consider frequency non-selective fast Rayleigh fading channel with relative velocity between the mobile transceivers set to 140 $\mathrm{km} / \mathrm{hr}$, carrier frequency $=2 \mathrm{GHz}$ and channel sampling time, $T_{s}=1 \mu \mathrm{s}$. This corresponds to a maximum doppler frequency, $f_{d}=259 \mathrm{~Hz}$ and results in channel decorrelation by a factor of 0.61 within a transmission block of 400 symbols. The adaptive nature, data resuse and symbol based processing of the algorithm enables to handle such fast fading. The results are averaged over 1000 simulation runs for each value of SNR in $\mathrm{dBs}$. Each simulation generates independent realization of Rayleigh fading channel with timing offset uniformly distributed as $\left(|\tau| \leq \frac{1}{2}\right) . N_{f}$ and $N$ are set to 2 for this simulation due to frequency non-selectivity. (a)

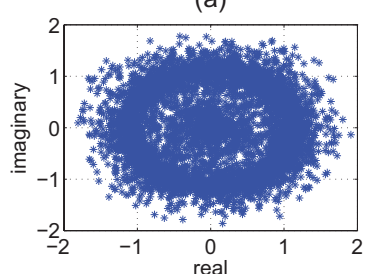

(c)
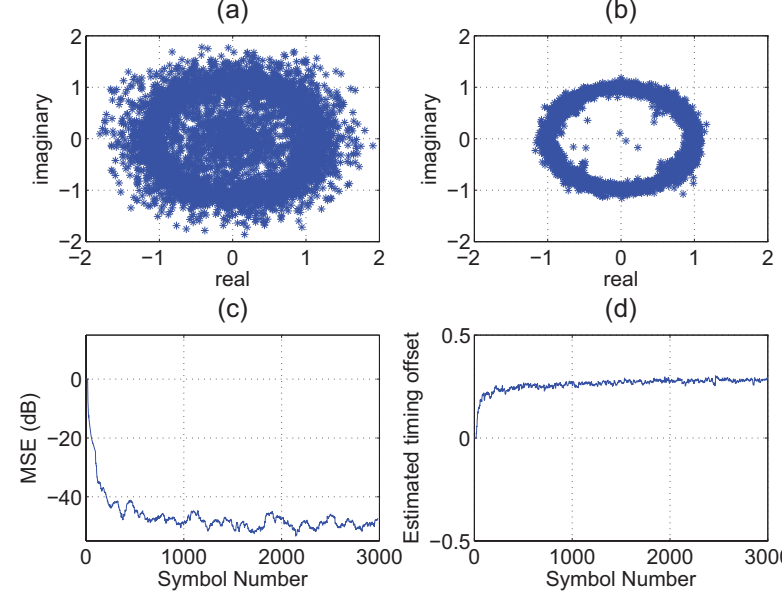

(d)

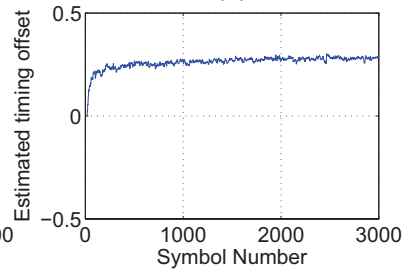

Fig. 3. Results for FSE with multipath channel, fractional timing phase offset $(\tau)=-0.3$, frequency offset $\left(\triangle f / F_{s}\right)=10^{-2}$, SNR $=20 \mathrm{~dB}$, DQPSK and equalizer length $N_{f}=7$. (a) Channel output constellation. (b) Equalized output constellation. (c) MSE between equalizer output and transmitted symbols. (d) Timing offset recovery.

Fig. 4 shows the BER results for Rayleigh fading channel for differential BPSK and QPSK modulation schemes with both TSE and FSE. FSE demonstrate approximately $2 \mathrm{~dB}$ gain in performance compared to TSE for higher values of SNR due to the advantages of FSE as mentioned in Sec III. Our results are just within 2 to $4 \mathrm{~dB}$ performance loss compared to the theoretical curves for DBPSK and DQPSK respectively. This small performance loss is due to the self noise of equalizer and timing offset estimation.

3) SUI Channels: We use SUI channels which are relevant to IEEE 802.16.3c standard for Wireless Metropolitan Area Networks [17]. We generate SUI-1,SUI-2 and SUI-3 channels, which are 3-ray Rician channel models with specific Rician $K$-factor, $K$, average path gains, $P$, and delay spread, $\tau_{d}$, for each tap. They are summarized in Table I [18]. We use DQPSK

TABLE I

SUI CHANNEL PARAMETERS

\begin{tabular}{c|c|c|c||c|c|c||c|c|c}
\hline & \multicolumn{3}{|c||}{ SUI-1 } & \multicolumn{3}{c||}{ SUI-2 } & \multicolumn{3}{c}{ SUI-3 } \\
\hline & $K$ & $\tau_{d}(\mu \mathrm{s})$ & $P(\mathrm{~dB})$ & $K$ & $\tau_{d}(\mu \mathrm{s})$ & $P(\mathrm{~dB})$ & $K$ & $\tau_{d}(\mu \mathrm{s})$ & $P(\mathrm{~dB})$ \\
\hline tap-1 & 4 & 0 & 0 & 2 & 0 & 0 & 1 & 0 & 0 \\
\hline tap-2 & 0 & 0.4 & -15 & 0 & 0.4 & -12 & 0 & 0.4 & -5 \\
\hline tap-3 & 0 & 0.9 & -20 & 0 & 1.1 & -15 & 0 & 0.9 & -10 \\
\hline
\end{tabular}

modulation scheme and $T_{s}=0.1 \mu$ s to model frequency selective channel dispersion up till 10 samples, so $N_{f}$ and $N$ are set to 10 for this simulation. The equalizer taps are initialized in the same manner as mentioned in Sec. IV-A. The system under such conditions can provide data rate of $5 \times 10^{6}$ symbols/sec, while using baseband bandwidh of $3.125 \mathrm{MHz}$. Results are simulated over 1000 different realizations for each SNR.

Fig. 5 shows the BER performance versus $E_{b} / N_{o}$ for FSE and TSE respectively. The theoretical BER results for Differential QPSK in selected SUI channels are used as reference. We can see that SUI-3 exhibits the worst BER results due 


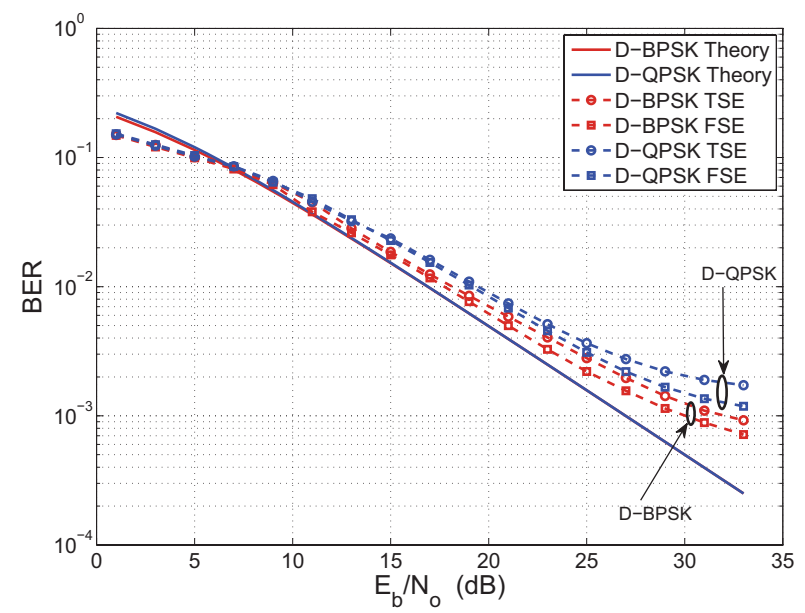

Fig. 4. BER versus $E_{b} / N_{o}(\mathrm{~dB})$ for time selective Rayleigh fading channel with doppler $f_{d}=259 \mathrm{~Hz}$, Sampling Time $T_{s}=1 \mu \mathrm{s}$, FSE length $N_{f}=2$, TSE length $N=2$ and Differential BPSK and QPSK modulations.

to strong multipath (power in tap-2 and tap-3) and small $K$-factor. Also SUI-1 has the best BER results because of dominant line of sight path $(K$-factor $=4)$ and weak ISI. From the figure, we can see 1.5 to $2 \mathrm{~dB}$ gain in performance for FSE compared to TSE at high SNR values for all SUIchannels, therefore, validating the system model. Moreover, all results meet the theoretical bounds with few $\mathrm{dB}$ acceptable loss in performance due to the self noise of equalizer and timing offset estimation. Thus proposed receiver is suitable for use in single source to destination link in wireless networks.

\section{CONCLUSiOns}

In this paper, we have accomplished joint blind equalization and timing recovery in fast-fading time and frequency selective wireless communication channels. We have proposed a system model for CMA based FSE with joint equalization and timing recovery and shown almost 2 times faster MSE convergence than symbol spaced equalization TSE. Our results have demonstrated that FSE outperforms TSE with approximately $2 \mathrm{~dB}$ gain in BER performance. We have achieved short burst wireless communication in CMA based equalization systems by proposing data reuse strategy. We have shown that our BER results meet the theoretical bounds with only a few $\mathrm{dB}$ loss in performance loss under severe time and frequency selective Rayleigh and Rician fading channels. Moreover, we have shown acceptable BER results in wireless Stanford University Interim (SUI) channels, which are relevant to IEEE 802.16.3c standard for Wireless Metropolitan Area Networks.

\section{REFERENCES}

[1] Z. Ding and Y. Li, Blind Equalization and Identification, K. J. Ray, Ed. Signal Processing and Communication Series, 2001.

[2] C. Y. Chi, C. C. Feng, C. H. Chen, and C. Y. Chen, Blind Equalization and System Identification. Springer, 2006.

[3] D. Ashmawy, K. Banovic, E. A. Raheem, M. Youssif, H. Manour, and M. Mohanna, "Joint MCMA and DD blind equalization algorithm with variable-step size," in IEEE International Conf. on Information Tech., 2009, pp. 174-177.

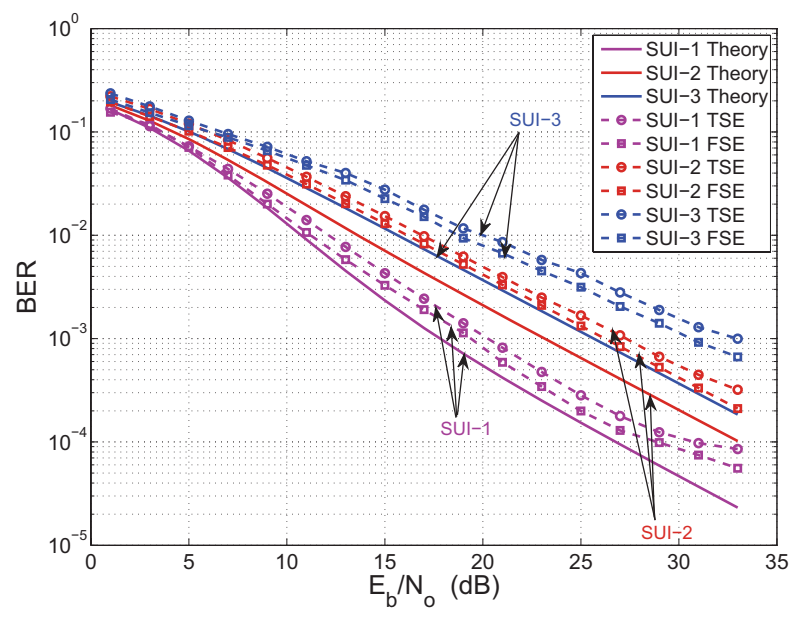

Fig. 5. BER versus $E_{b} / N_{o}(\mathrm{~dB})$ for SUI fading channels with Sampling Time $T_{s}=0.1 \mu \mathrm{s}$, FSE length $N_{f}=10$, TSE length $N=10$ and Differential QPSK modulation.

[4] Y. Zhang, J. Zhao, J. Li, and Y. Sun, "A fast convergent algorithm for joint blind equalization and carrier recovery," in Proc. ICSP, 2008, pp. 1784-1787.

[5] A. A. Nasir, S. Durrani, and R. A. Kennedy, "Modified Constant Modulus Algorithm for joint blind equalization and synchronization,' in Proc. AusCTW, Feb. 2010

[6] V. Sharma and V. N. Raj, "Convergence and performance analysis of godard family and multimodulus algorithms for blind equalization," IEEE Trans. Signal Process., vol. 53, no. 4, pp. 1520-1533, Apr. 2005.

[7] D. N. Godard, "Self-recovering equalization and carrier tracking in twodimensional data communication systems," IEEE Trans. Commun., vol. COM-28, pp. 1867-1875, 1980.

[8] Y. Li and Z. Ding, "Global convergence of fractionally spaced Godard (CMA) adaptive equalizers," IEEE Trans. Signal Process., vol. 44, no. 4, pp. 818-826, April 1996.

[9] I. Fijalkow, A. Touzni, and J. R. Treichler, "Fractionally spaced equalization using CMA: Robustness to channel noise and lack of disparity," IEEE Trans. Signal Process., vol. 45, no. 1, pp. 56-66, Jan. 1997.

[10] T. Ohgane, H. Sasaoka, N. Matsuzawa, and T. Shimura, "BER performance of CMA adaptive array for high-speed GMSK mobile communication - a description of measurements in central tokoyo," IEEE Trans. Veh. Technol., vol. 42, pp. 484-490, Nov. 1993.

[11] B. J. Kim and D. C. Cox, "Blind equalization for short burst wireless communications," IEEE Trans. Veh. Technol., vol. 49, no. 4, pp. 1235 1247, Jul. 2000.

[12] G. Picchi and G. Prati, "Blind equalization and carrier recovery using a "Stop-and-Go" decision directed algorithm," IEEE Trans. Commun., vol. COM-35, pp. 877-887, Sep 1987.

[13] J. Proakis, Digital Communications, 4th ed. New York: McGraw-Hill, 2000

[14] Z. Ding, R. A. Kennedy, D. O. Anderson, and J. C. R. Johnson, "Ill-convergence of Godard blind equalizers in data communication systems," IEEE Transactions on Communications, vol. 39, pp. 1313 1327, Sept. 1991.

[15] H. Meyr, M. Moeneclaey, and S. A. Fechtel, Digital Communication Receivers, Synchronization, Channel Estimation, and Signal Processing. Wiley Series in Telecom. and Signal Processing, 1998.

[16] I. Santamaria, C. Pantaleon, L. Vielva, and J. C. Principle, "A fast algorithm for adaptive blind equalization using order-a renyi's entropy," in IEEE Conf. on Acoustics, Speech and Signal Processing, vol. 13, no. 2, 2002, pp. 2657-2660.

[17] IEEE 802.16 standard for Wireless Metropolitan Area Networks [Online]. Available: http://www.ieee802.org/16/

[18] Channel models for fixed wireless applications. [Online]. Available: http://wirelessman.org/tga/docs/80216a-03_01.pdf 